\title{
Dynamic changes of serum SARS-Coronavirus IgG, pulmonary function and radiography in patients recovering from SARS after hospital discharge

\author{
Lixin Xie ${ }^{1}$, Youning Liu*1, Baoxing Fan1, Yueyong Xiao², Qing Tian¹, \\ Liangan Chen ${ }^{1}$, Hong Zhao ${ }^{2}$ and Weijun Chen ${ }^{3}$
}

Address: ${ }^{1}$ Department of Respiratory Medicine, Chinese PLA General Hospital, 28 Fuxing Road, Beijing, 100853, P.R. China, ${ }^{2}$ Department of Radiology, Chinese PLA General Hospital, 28 Fuxing Road, Beijing, 100853, P.R. China and 3BGI-GBI Biotech Company, Beijing, P.R. China

Email: Lixin Xie - xielx@301hospital.com.cn; Youning Liu* - liuyn@301hospital.com.cn; Baoxing Fan - fanbx@263.net.cn;

Yueyong Xiao - xiaoyuryong@vip.sina.com; Qing Tian - tianqing777@sohu.com; Liangan Chen - chenliangan@301hospital.com.cn; Hong Zhao - liuyn@301hospital.com.cn; Weijun Chen - chenweijun72@sohu.com

* Corresponding author

Published: 08 January 2005

Respiratory Research 2005, 6:5 doi:10.1 186/1465-9921-6-5

This article is available from: http://respiratory-research.com/content/6/1/5

(c) 2005 Xie et al; licensee BioMed Central Ltd.

This is an Open Access article distributed under the terms of the Creative Commons Attribution License (http://creativecommons.org/licenses/by/2.0), which permits unrestricted use, distribution, and reproduction in any medium, provided the original work is properly cited.

\begin{abstract}
Objective: The intent of this study was to examine the recovery of individuals who had been hospitalized for severe acute respiratory syndrome (SARS) in the year following their discharge from the hospital. Parameters studied included serum levels of SARS coronavirus (SARS-CoV) IgG antibody, tests of lung function, and imaging data to evaluate changes in lung fibrosis. In addition, we explored the incidence of femoral head necrosis in some of the individuals recovering from SARS.

Methods: The subjects of this study were 383 clinically diagnosed SARS patients in Beijing, China. They were tested regularly for serum levels of SARS-CoV IgG antibody and lung function and were given chest $X$-rays and/or high resolution computerized tomography (HRCT) examinations at the Chinese PLA General Hospital during the 12 months that followed their release from the hospital. Those individuals who were found to have lung diffusion abnormities (transfer coefficient for carbon monoxide $\left[D_{L} C O\right]<80 \%$ of predicted value [pred]) received regular lung function tests and HRCT examinations in the follow-up phase in order to document the changes in their lung condition. Some patients who complained of joint pain were given magnetic resonance imaging (MRI) examinations of their femoral heads.

Findings: Of all the subjects, $81.2 \%$ ( 3 II of 383 patients) tested positive for serum SARS-CoV IgG. Of those testing positive, $27.3 \%$ (85 of 31 I patients) were suffering from lung diffusion abnormities $\left(D_{1} C O<80 \%\right.$ pred) and $21.5 \%$ (67 of 3 II patients) exhibited lung fibrotic changes. In the 12 month duration of this study, all of the 40 patients with lung diffusion abnormities who were examined exhibited some improvement of lung function and fibrosis detected by radiography. Of the individuals receiving MRI examinations, $23.1 \%$ (18 of 78 patients) showed signs of femoral head necrosis.

Interpretation: The lack of sero-positive SARS-CoV in some individuals suggests that there may have been some misdiagnosed cases among the subjects included in this study. Of those testing positive, the serum levels of SARS-CoV IgG antibody decreased significantly during the 12 months after hospital discharge. Additionally, we found that the individuals who had lung fibrosis showed some spontaneous recovery. Finally, some of the subjects developed femoral head necrosis.
\end{abstract}




\section{Introduction}

Severe acute respiratory syndrome (SARS) is a new infectious disease in humans. The first victim of SARS to be diagnosed was a businessman from the city of Foshan in Guangdong Province, China. SARS patients may present with a spectrum of symptoms and signs, ranging from relatively asymptomatic to fulminant pneumonitis and death [1]. Lung injury caused by the SARS coronavirus (SARS-CoV) is one of the main clinical manifestations in SARS patients, significantly affecting their prognosis. A regular follow-up survey of SARS patients in the convalescent phase would be helpful to evaluate any changes in acquired immune function, pulmonary function, bones and joints over the course of time. At present, there have been few reports about the relationship between the prognosis for recovery and the degree of lung injury caused by the SARS-CoV. In addition, a study of the serum levels of the specific IgG antibody against SARS-CoV is needed because it is the major immunologic protection to aid in recovery and is essential to avoid repeated infection with SARS-CoV. It has been 14 months since the World Health Organization officially declared the global outbreak of SARS to be under control [2]. The present study focused on the dynamic changes in the IgG antibody levels against SARS-CoV and in lung lesions in the discharged but recovering SARS patients as measured by lung function and imaging tests. The phenomenon of femoral head necrosis was also investigated in those SARS patients who complained of chronic bone and joint pain during the one year follow-up after discharge from the hospital.

\section{Methods}

All of the subjects of this study were discharged from Beijing Xiaotangshan Hospital, Beijing Armed Police Hospital, and Chinese 309 PLA Hospital, and all gave their informed consent.

\section{Study Protocol}

The subjects of our investigation were 383 clinically diagnosed SARS patients in the convalescent phase (160 male and 223 female, average age $38.2 \pm 13.6$ years) undergoing testing from May, 2003 to June, 2004. Each clinical diagnosis was based on the Clinical Diagnosis Standard for SARS Patients issued by the Ministry of Chinese Public Health [3]. All participants in the study had met the specified criteria for discharge from the hospital [4]. On the first visit, each patient was given a routine pulmonary function test (ventilation and diffusion function: SensorMedics 2200 pulmonary function test apparatus, U.S.A.), a chest X-ray examination and serum SARS-CoV specific antibody (SARS-CoV IgG) test at the Chinese PLA General Hospital, Beijing, P.R. China. Those individuals suspected of having pulmonary fibrotic changes received high resolution computerized tomography (HRCT) examination of their lungs. Individuals who complained of chronic pain in their bones and joints or who had difficulty walking received femoral head magnetic resonance imaging (MRI) examinations.

Each patient returned a month after the first visit followed by one visit every 3 months. Serum SARS-CoV IgG was tested at each return visit. If negative results were obtained twice consecutively, the case was regarded as a misdiagnosis and the patient did not undergo a follow-up survey. Patients with positive SARS-CoV IgG and abnormal pulmonary diffusion received regular pulmonary function tests and those showing pulmonary fibrosis in imaging examinations received further regular HRCT examinations. Some individuals observed to have avascular necrosis of the femoral head received MRI examinations 3-6 months later.

\section{Clinical Diagnostic Criteria for the Patients with SARS Disease in Mainland China [3]}

(I) Epidemiological history

(1.1) The individual has a history of close contact with SARS patients or is part of a cluster of cases of SARS or there is clinical evidence of having infected other patients.

(1.2) The individual has a history of recent travel to an area where SARS cases have been reported within 2 weeks and secondary infected SARS cases have been found.

\section{(2) Symptoms and signs}

Acute onset of SARS generally begins with a prodrome of fever with a temperature $>38^{\circ} \mathrm{C}$, sometimes accompanied by chills, myalgia and anthralgia, headaches, and fatigue. Upper respiratory tract symptoms of catarrh are not prominent, although cough may be present. If present, it is mainly a dry cough, occasionally with blood streak sputum. Some individuals have chest discomfort, and severe cases may present with tachypnea, panting, and even respiratory distress.

Generally, there are no obvious pulmonary signs among SARS patients. Wet rales and signs of lung consolidation, as well as decreased respiration and other signs of pleural effusion can occasionally be found.

Note: Some patients do not show initial symptoms of fever, especially those who have had recent surgery or those having chronic diseases.

\section{(3) Routine laboratory examinations}

White blood cell counts are generally normal or below normal, with decreased absolute lymphocyte counts.

\section{(4) Chest radiological examinations}

The typical imaging profile of SARS is of multiple patchy opacities with bilateral distribution. The opacities are 
usually ground-glass in appearance, sometimes with air space consolidation, evolving progressively over the course of the disease. The evolution is very rapid in some cases, resulting in the confluence of lesions and large areas of opacification in a short time. If the chest radiological examination is negative, reexamination after 1 to 2 days should be done.

\section{(5) Antibiotic therapy is ineffective}

Suspected cases: In accordance with $1.1+2+3,1.2+2+4$ or $2+3+4$.

Clinically diagnosed cases: In accordance with $1.1+2+4$, $1.2+2+4+5$, or $1.2+2+3+4$.

\section{SARS-CoV IgG Antibody Test}

The SARS-CoV IgG antibody in serum specimens from recovering SARS patients was assayed by the BGI-GBI Biotech Company with an enzyme-linked immunosorbent assay (ELISA) kit (No. S20030003, BGI-GBI Biotech Company, Beijing, P.R. China). The wells containing polystyrene microplate strips were coated with two recombinant SARS-CoV antigens that are well-characterized. Recovering SARS patients' serum samples in the diluent buffer $(1: 10)$ were incubated in the coated wells for $30 \mathrm{~min}$ at $37^{\circ} \mathrm{C}$ and then the wells were washed 5 times with the washing buffer. The dilutedenzyme-labeled anti-human IgG $(100 \mu \mathrm{l})$ was added to the wells and incubated for 20 $\min$ at $37^{\circ} \mathrm{C}$. The wells were washed 5 times with the washing buffer. A tetramethyl-benzidine substrate was then added to each well. The presence of specific antibodies was indicated by a yellow color developing after substrate addition. The reaction was terminated by addition of hydrochloric acid. The intensity of the color was measured spectrophotometrically at $450 \mathrm{~nm}$ to quantify the amount of antibody in the specimen. The optical density (OD) measured was compared with a standard calibration curve constructed for each lot, yielding concentration values for the samples. The OD values of both the positive and negative controls were determined. The threshold value for IgG was 0.18 OD units, calculated as the mean + 2 standard deviation (SD) levels of the readings given by 1000 control blood donor sera samples. If the OD was above the threshold value, the sample was considered to be positive for SARS-CoV IgG [5].

\section{Pulmonary Function Test}

Each recovering SARS patient underwent a standard pulmonary function test (SensorMedics 2200, Yorba Linda, U.S.A) for forced expiratory volume in 1 second $\left(\mathrm{FEV}_{1}\right)$, vital capacity (VC), forced vital capacity (FVC), total lung capacity (TLC), transfer coefficient for carbon monoxide $\left(\mathrm{D}_{\mathrm{L}} \mathrm{CO}\right)$, and carbon monoxide diffusion constant $\left(D_{L} C O / V_{A}\right)$ measured by means of the single-breath test. The hemoglobin level was also measured to adjust the
$D_{L} C O$ value. The results were compared with those of ageand sex-matched controls and expressed as a percentage of predicted values. Pulmonary function was regarded as abnormal when the $\mathrm{D}_{\mathrm{L}} \mathrm{CO}$ was less than $80 \%$ of predicted values (pred). This was considered a diffusion deficit [6].

\section{Chest Radiography and Evaluation}

Frontal chest X-ray radiographs (CXR) were obtained at the first follow-up visit for each recovering SARS patient. If abnormities were found in the CXR or if the $\mathrm{D}_{\mathrm{L}} \mathrm{CO}$ was $<80 \%$ pred despite a normal CXR, the patient was sent for HRCT scanning (GE Light Speed, GE, U.S.A. 1-mm section in thickness with a 10-mm gap, supine position, scanning during inspiration, 1 second per scan, $140 \mathrm{kv}, 200 \mathrm{~mA}$ ). All CXR and HRCT images were assessed by three radiologists via a viewing console. The three radiologists were aware of the patients' clinical diagnosis at the time of their review of the radiographs. The final conclusions were established by consensus. Each segment of the lung was reviewed for ground-glass opacification, interstitial thickening, bronchiectasis, and architectural distortion. Abnormalities were magnified by means of a zoom function and were examined for intralobular interstitial, interlobular septal, or peribronchovascular interstitial thickening. Attention was also paid to the presence or absence of nodules or masses, cavitation or calcification, and emphysema. The presence of parenchymal bands, irregular interfaces (bronchovascular, pleural, or mediastinal), thickened interstitium, and traction bronchiectasis were considered as evidence of fibrotic changes [7].

\section{Magnetic Resonance Imaging (MRI) Examination}

All MRI examinations were done using a $1.5 \mathrm{~T}$ Signa CVi imager (GE Medical Systems, Milwaukee, WI, U.S.A.). For the patients who complained of chronic bone and joint pain, coronal $\mathrm{T}_{1}$-weighted (spin echo; time to repetition [TR], 440-500; time to echo [TE) 11-14] scans of the hips were done. If there were any abnormalities noted in the $\mathrm{T}_{1}$-weighted images, further $\mathrm{T}_{1}$-weighted sagittal images and coronal short tau inversion recovery (inversion time 145, TR 3500-5000, TE 80-120) or turbo-spin-echo $\mathrm{T}_{2}$ weighted images with fat suppression (TR 2500-3000, TE 80-120) were obtained. Images $3 \mathrm{~mm}$ thick were obtained for the coronal studies, and $4 \mathrm{~mm}$ thick images were obtained for the sagittal studies. Osteonecrosis was diagnosed by the presence of a band of low signal intensity in $\mathrm{T}_{1}$-weighted images [8].

\section{Statistical Analysis}

All data were expressed as the $\bar{X} \pm$ SD unless otherwise indicated. Statistical analyses were done by one-way analysis of variance (ANOVA), Student-Newman-Keuls, and Chi-square test for multiple comparisons. We used the STATA $^{\text {тм }} 7.0$ statistical analysis software for Windows (STATA Statistical Software, Inc., U.S.A.) for evaluating the 
Table I: Dynamic changes of serum SARS-CoV IgG antibody levels in patients recovering from SARS

\begin{tabular}{lll}
\hline & Samples $(\mathrm{n})$ & $\bar{X} \pm$ SD (OD units) \\
\hline May, 2003 & 35 & $1.240 \pm 0.350$ \\
June, 2003 & 74 & $1.087 \pm 0.284$ \\
July, 2003 & 172 & $1.203 \pm 0.306$ \\
Aug., 2003 & 152 & $1.061 \pm 0.376$ \\
Sept., 2003 & 123 & $1.105 \pm 0.378$ \\
Oct., 2003 & 35 & $1.097 \pm 0.282$ \\
Nov., 2003 & 77 & $0.835 \pm 0.327 \dagger \ddagger \S T^{*}$ \\
Dec., 2003 & 35 & $0.829 \pm 0.232+\S^{*}$ \\
Jan.-Feb., 2004 & 67 & $0.737 \pm 0.169 \dagger \ddagger \S T^{*} \#$ \\
Mar-Apr, 2004 & 34 & $0.678 \pm 0.179 \dagger \ddagger \S T^{*} \#$ \\
May-June, 2004 & 46 & $0.621 \pm 0.181 \dagger \ddagger \S T^{*} \#$ \\
F value & & 30.62 \\
P value & & 0.0000 \\
\hline
\end{tabular}

Note: Statistical analyses were done by one-way analysis of variance (ANOVA) and Student-Newman-Keuls for multiple comparisons, and values are given as mean $\pm S D$;

$\dagger p<0.05$ vs. SARS-CoV IgG antibody results in May, 2003.

$\ddagger p<0.05$ vs. SARS-CoV IgG antibody results in June, 2003.

$\S p<0.05$ vs. SARS-CoV IgG antibody results in July, 2003.

II $p<0.05$ vs. SARS-CoV IgG antibody results in August, 2003.

$* p<0.05$ vs. SARS-CoV IgG antibody results in Sepember, 2003.

$\# p<0.05$ vs. SARS-CoV IgG antibody results in October, 2003.

Table 2: Dynamic changes of serum SARS-CoV IgG antibody levels in 33 regular follow-up examinations of patients recovering from SARS

\begin{tabular}{lll}
\hline & Samples $(\mathrm{n})$ & $\bar{X} \pm$ SD (OD units) \\
\hline June, 2003 & 33 & $1.104 \pm 0.267$ \\
July, 2003 & 33 & $1.325 \pm 0.357$ \\
Aug., 2003 & 33 & $1.092 \pm 0.249$ \\
Sept., 2003 & 33 & $1.121 \pm 0.432$ \\
Oct., 2003 & 33 & $1.056 \pm 0.309$ \\
Nov., 2003 & 33 & $0.895 \pm 0.203 \ddagger \pi$ \\
Dec., 2003 & 33 & $0.800 \pm 0.170 \dagger \ddagger \S \pi$ \\
Jan.-Feb., 2004 & 33 & $0.726 \pm 0.163 \dagger \ddagger \S T^{*}$ \\
Mar-Apr, 2004 & 33 & $0.675 \pm 0.181 \dagger \ddagger \delta T^{*}$ \\
May-June, 2004 & 33 & $0.610 \pm 0.167 \dagger \ddagger \S T^{*} \#$ \\
F value & & 25.69 \\
P value & & 0.0000
\end{tabular}

Note: Statistical analyses were done by one-way analysis of variance (ANOVA) and Student-Newman-Keuls for multiple comparisons, and values are given as mean $\pm S D$;

$t p<0.05$ vs. SARS-CoV IgG antibody results in June, 2003.

$\ddagger p<0.05$ vs. SARS-CoV IgG antibody results in July, 2003.

$\S p<0.05$ vs. SARS-CoV IgG antibody results in August, 2003.

II $p<0.05$ vs. SARS-CoV IgG antibody results in September, 2003.

$* p<0.05$ vs. SARS-CoV lgG antibody results in October, 2003.

$\# p<0.05$ vs. SARS-CoV IgG antibody results in November, 2003. results of our study. With each statistical test, the criterion for significance was a $p$ value of less than 0.05 .

\section{Results}

The interval from hospital discharge to the first follow-up visit was $45.0 \pm 20.7$ days (Range: 11-104 days). Of the 383 individuals participating in our study, 311 patients (81.2\%) tested positive for SARS-CoV IgG and 72 (18.8\%) tested negative. (All patientswere tested twice for SARSCoV IgG.) Of these, 33 patients (13 male and 20 female, average age $35.7 \pm 12.1$ ) with positive SARS-CoV IgG and abnormal pulmonary diffusion received regular follow-up examinations each month, from June to December in 2003, and every two months, from January to June in 2004. Tables 1 and 2 show the dynamic changes of SARSCoV IgG in patients with positive tests for SARS-CoV IgG within the year after discharge, indicating that the serum SARS-CoV IgG remained at high levels, although it decreased significantly over the course of time.

There were 88 individuals $(23.0 \%)$ with abnormal $\mathrm{D}_{\mathrm{L}} \mathrm{CO}$ among the 383 patients participating in our study. Of the 311 individuals testing positive for SARS-CoV IgG, there were 85 with abnormal $\mathrm{D}_{\mathrm{L}} \mathrm{CO}(27.3 \%, 85 / 311)$, in contrast to just 3 cases with abnormal $\mathrm{D}_{\mathrm{L}} \mathrm{CO}$ among the 72 subjects testing negative for SARS-CoV IgG $(4.2 \%, 3 / 72)$. There was a statistically significant difference between positive and negative SARS-CoV IgG groups in $\mathrm{D}_{\mathrm{L}} \mathrm{CO}$ values (table 3).

Among the 85 patients (29 male and 56 female, average age $42.2 \pm 11.9$ years) with abnormal $\mathrm{D}_{\mathrm{L}} \mathrm{CO}$ and positive SARS-CoV IgG, 40 individuals received pulmonary function tests 4 times within the year at $42.0 \pm 10.4,70.0 \pm$ 11.8 and $155.1 \pm 42.9$ day intervals. Among these 40 patients, there were 23 who exhibited abnormal $\mathrm{D}_{\mathrm{L}} \mathrm{CO}$ at their second pulmonary function examination, 23 at the third examination, and 20 at the fourth examination (table 4).

Pulmonary fibrosis was detected by CXR and confirmed by HRCT examination in 72 SARS patients in the convalescent phase. Among these, there were 4 individuals with negative and 67 with positive SARS-CoV IgG. Of the 40 patients who received HRCT examinations at least 4 times, all showed improvement in the fibrotic condition (Figure 1).

Of the 311 convalescent SARS patients with sero-positive SARS-CoV IgG, 78 received femoral head MRI examinations. The Imaging showed that 18 of these patients $(23.1 \%, 18 / 78)$ had avascular necrosis of the femoral head. Of these 18 individuals, 8 had avascular necrosis of 


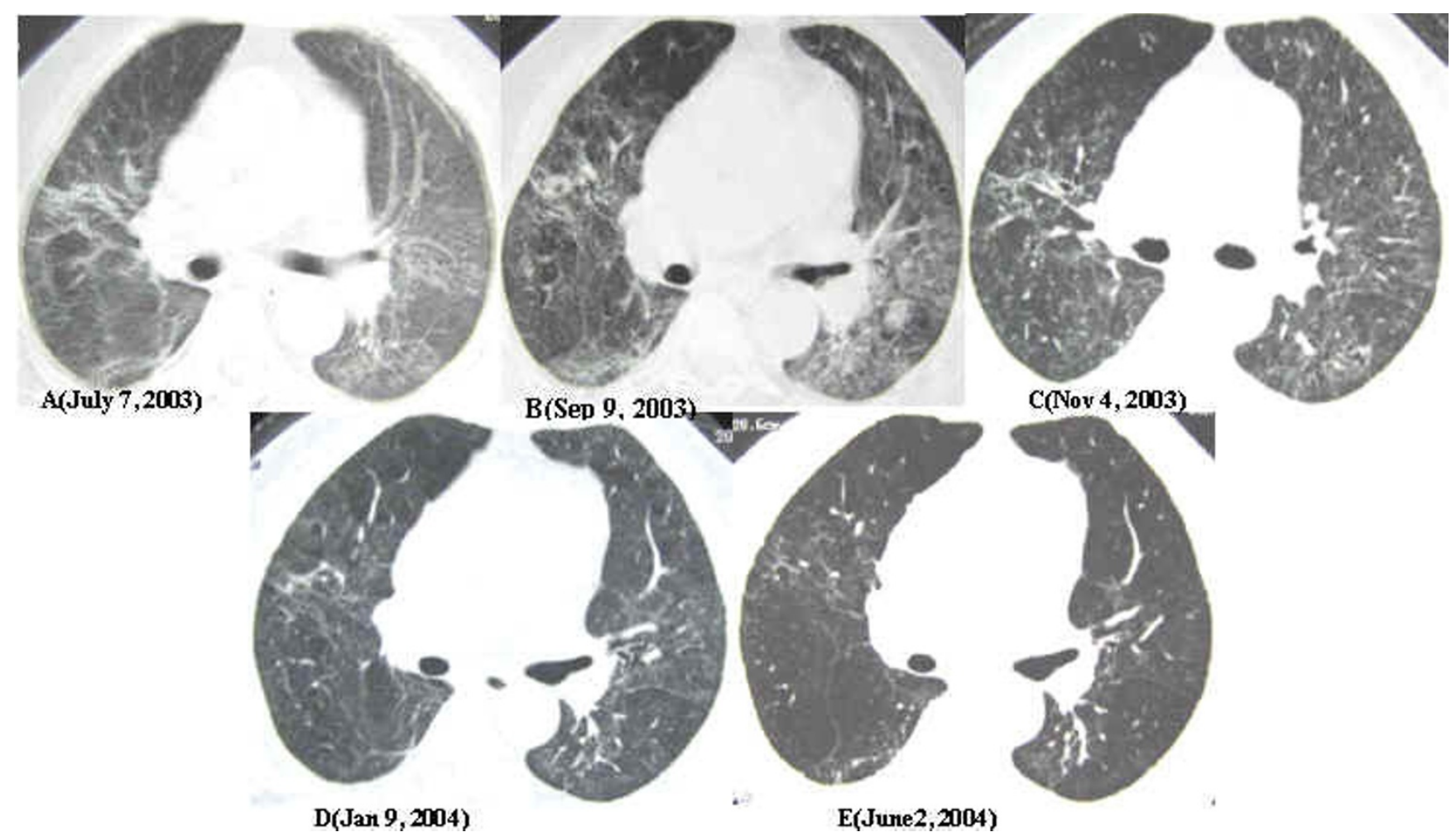

Figure I

The results of chest HRCT examination in a SARS patient in the convalescent phase, showing marked reversal of pulmonary fibrosis.

both femoral heads and 10 had avascular necrosis of one femoral head. Ten of the 18 patients showed first stage changes and 8 had secondary changes. During the 3-6 month follow-up visits for these individuals, there were no obvious changes in the avascular necrosis for these patient.

\section{Discussion}

Since the outbreak of SARS at the end of 2002, despite the great efforts that have been extended, the mechanisms, clinical characteristics, prognosis and effective therapeutics for this disease have not been adequately clarified. Both the SARS virus itself and the anti-viral therapy (such as high-dose glucocorticoids) used in treatment can cause various degrees of toxicity and side effects, including pulmonary fibrosis and avascular necrosis of the femoral head, even in the convalescent phase. Follow-up surveys of SARS patients in the convalescent phase are needed for recognizing the clinical characteristics of this disease and reevaluation of the therapeutic treatments $[2,7]$.

In our study, 72 individuals (18.8\%) showed negative results in the SARS-CoV IgG antibody test for at least two tests, suggesting that there may have been misdiagnosis of some clinically diagnosed SARS patients. Comparison of the Chinese clinical diagnosis standard (published April, 2003) [3] to the Center for Disease Control (CDC) SARS case definition (published April 30, 2003) [9], indicates that both of them emphasize the importance of epidemiological history, clinical manifestations and chest radiological changes for the clinical diagnosis of SARS disease. The CDC SARS case definition especially emphasizes the importance of laboratory criteria for the confirmation of a SARS diagnosis. This is accomplished by detecting the dynamic changes in the titration of specific antibodies against SARS CoV and positive detection of SARS-CoV RNA by PCR. In contrast, the Chinese clinical diagnosis standard did not mention the importance of a laboratory SARS-CoV test for the confirmation of a SARS diagnosis. This might have resulted in the misdiagnosis of SARS in some cases. During follow-up examinations, we found that those individuals with positive SARS-CoV IgG remained positive for a year, although the level of the antibody decreased gradually. Therefore, those inoculated with a SARS vaccine or infected by the SARS virus might not receive lifetime immunity, but only immunity for a 
Table 3: $D_{L} C O$ results for the convalescent SARS patients with sero-positive or sero-negative SARS-CoV IgG

\begin{tabular}{llllll}
\hline & Positive & Negative & Total & $X^{2}$ & $P$ value \\
\hline$D_{\mathrm{L}}$ CO normal cases & 226 & 69 & 295 & & \\
$\mathrm{D}_{\mathrm{L}} \mathrm{CO}$ abnormal cases & 85 & 3 & 88 & 17.7269 & 0.000 \\
Total & 311 & 72 & 383 & & \\
\hline
\end{tabular}

Note: Analyzed with Chi-square test.

Table 4: Pulmonary function test results from the 4 follow-up examinations of 40 convalescent SARS patients ( $\bar{X} \pm$ SD)

\begin{tabular}{lllll}
\hline Follow-up* & $\mathrm{VC}(\%$ pred $)$ & $\mathrm{FEV}(\%$ pred $)$ & $\mathrm{D}_{\mathrm{L}} \mathrm{CO}(\%$ pred $)$ & $\mathrm{D}_{\mathrm{L}} \mathrm{CO} / \mathrm{V}_{\mathrm{A}}(\%$ pred $)$ \\
\hline Two months & $87 \pm 15(51 \sim 114)$ & $83 \pm 13(60 \sim 108)$ & $69 \pm 9(47 \sim 79)$ & $95 \pm 14(58 \sim 123)$ \\
Four months & $94 \pm 14(61 \sim 123)$ & $90 \pm 13(65 \sim 121)$ & $76 \pm 11(48 \sim 94)$ & $99 \pm 14(67 \sim 126)$ \\
Six months & $100 \pm 15 \dagger(66 \sim 136)$ & $93 \pm 12 \dagger(66 \sim 114)$ & $76 \pm 11 \dagger(52 \sim 98)$ & $97 \pm 14(62 \sim 129)$ \\
Eleven months & $103 \pm 15 \dagger(66 \sim 142)$ & $96 \pm 11 \dagger(67 \sim 115)$ & $79 \pm 12 \dagger(56 \sim 98)$ & $97 \pm 14(59 \sim 128)$ \\
$F$ value & 9.23 & 7.84 & 6.15 & 0.63 \\
$P$ value & 0.0000 & 0.0001 & 0.0006 & 0.5936
\end{tabular}

Note: *: Indicating as time after discharge from acute illness.

Statistical analyses were done by one-way analysis of variance (ANOVA) and Student-Newman-Keuls for multiple comparisons, and values are given as mean $\pm \mathrm{SD}$;

$\dagger$ : Compared to those in the first follow-up exam, $p<0.05$.

limited duration. Certainly, our findings must be confirmed by further studies $[7,8]$.

By regular examination of pulmonary function and CXR, we found that those with pulmonary fibrotic changes were able to heal on their own. The fibrotic tissue was absorbed and pulmonary diffusion and VC improved with time, suggesting that the mechanism of lung injury and lung fibrosis caused by the SARS-CoV may have a different pathophysiological process compared to other lung diseases, such as idiopathic pulmonary fibrosis or pulmonary fibrosis secondary to adult respiratory stress syndrome. The reason is not clear. However, in our follow-up study, we found some ground-glass-like changes in the HRCT images from SARS patients one year after discharge. This result shows that changes in the lung can still be observed in convalescents $[7,9]$.

Recent concern has focused on a complication of SARS in the convalescent phase, when avascular necrosis develops on the femoral head. The morbidity of this condition is reported to be $15 \%$ to $30 \%$ in some SARS patients in Mainland China $[8,10]$. Among the 78 patients receiving an MRI examination, there were 18 cases of complicated necrosis of the femoral head to different degrees. The causes of this complication include SARS itself and the drugs (such as glucocorticoids) used in treatment, with the latter being more important than the former [11-13].
We didn't find any worsening or improvement of the avascular necrosis of the femoral head in these patients during our follow-up examinations. Although most patients received magnetotherapy, hyperbaric oxygen chamber therapy, local kerotherapy and Chinese traditional medicine to promote local blood circulation, there was no apparent short-term therapeutic effectiveness of these methods for recovery of the femoral head.

In conclusion, SARS, as a new disease, remains unfamiliar to mankind. It has high rates of morbidity and mortality in the acute phase. A significant proportion of patients surviving the acute illness have impairment in their overall functional capacity and health status in the convalescent phase after discharge from the hospital. Follow-up surveys of SARS patients in the convalescent phase are needed to understand the clinical characteristics of this disease. Our findings suggest that follow up studies of these patients are required for a longer duration, including comprehensive assessments for detection and appropriate management of any persistent or emerging sequelae. These types of investigation may facilitate the search for effective therapeutics and aid in ultimately conquering this disease.

\section{Acknowledgments}

We are thankful for the research funding from the National High Technology Research and Development Program of China (863 Program) 
2003AA208107 from Ministry of Science and Technology, the People's Republic of China.

\section{References}

I. Chinese Respiratory Association: Consensus for the diagnosis standard and management of severe acute respiratory syndrome. Zhonghua Jie He He Hu Xi Za Zhi 2003, 26:323-325.

2. Chinese Medical Association: Consensus for the management of severe acute respiratory syndrome. Chin Med J 2003, I 16: I603-1635.

3. Ministry of Chinese Public Health: Clinical diagnosis consulting criteria for the patients with severe acute respiratory syndrome disease (Apr 4, 2003). [http://www.moh.gov.cn/public/ open.aspx?n id=7310\&seq=0].

4. Ministry of Chinese Public Health: Diagnosis standard, treatment proposal and discharge consulting criteria for severe acute respiratory syndrome. [http://www.moh.gov.cn/public/ open.aspx? $n$ id $=7286 \&$ seq $=0]$.

5. Chen W, Xu Z, Mu J, Yang L, Gan H, Mu F, Fan B, He B, Huang S, You B, Yang Y, Tang X, Qiu L, Qiu Y, Wen J, Fang J, Wang J: Antibody response and viraemia during the course of severe acute respiratory syndrome (SARS)-associated coronavirus infection. J Med Microbiol 2004, 53:435-438.

6. Xie LX, Liu YN, Fan BX, Chen LA, Hao FY, Cao L, Tian Q, Ma L: Prognostic Analysis of Serum SARS-Co V IgG antibody, Lung function and Lung Radiographic Changes of SARS Patients in Six Months after Discharge. Chin PLA Med J 2004, 29:762-764.

7. Antonio GE, Wong KT, Hui DSC, Wu A, Lee N, Yuen EH, Leung CB, Rainer TH, Cameron P, Chung SS, Sung J], Ahuja AT: Thin-section $C T$ in patients with severe acute respiratory syndrome following hospital discharge: preliminary experience. Radiology 2003, 228:810-815.

8. Li YM, Wang SX, Gao HS, Wang JG, Wei CS, Chen LM, Hui WL, Yuan SL, Jiao ZS, Yang Z, Su B: Factors of avascular necrosis of femoral head and osteoporosis in SARS patients' convalescence. Zhonghua Yi Xue Za Zhi 2004, 84: I348-I 353.

9. Shichijo S, Keicho N, Long HT, Quy T, Phi NC, Ha LD, Ban VV, Itoyama S, Hu CJ, Komatsu N, Kirikae T, Kirikae F, Shirasawa S, Kaji M, Fukuda T, Sata M, Kuratsuji T, Itoh K, Sasazuki T: Assessment of synthetic peptides of severe acute respiratory syndrome coronavirus recognized by long-lasting immunity. Tissue Antigens 2004, 64:600-607.

10. Chan CW, Chiu WK, Chan CC, Chow EY, Cheung HM, Ip PL: Osteonecrosis in children with severe acute respiratory syndrome. Pediatr Infect Dis J 2004, 23:888-890.

II. Hong N, Du XK: Avascular necrosis of bone in severe acute respiratory syndrome. Clin Radiol 2004, 59:602-608.

12. Chan KS, Zheng JP, Mok YW, Li YM, Liu YN, Chu CM, Ip MS: SARS: prognosis, outcome and sequelae. Respirology 2003, 8(Suppl):S36-S40.

13. Koo KH, Kim R, Kim YS, Ahn IO, Cho SH, Song HR, Park YS, Kim H, Wang GJ: Risk period for developing osteonecrosis of the femoral head in patients on steroid treatment. Clin Rheumatol 2002, 2I:299-303.

\section{Publish with Bio Med Central and every scientist can read your work free of charge}

"BioMed Central will be the most significant development for disseminating the results of biomedical research in our lifetime. "

Sir Paul Nurse, Cancer Research UK

Your research papers will be:

- available free of charge to the entire biomedical community

- peer reviewed and published immediately upon acceptance

- cited in PubMed and archived on PubMed Central

- yours - you keep the copyright

Submit your manuscript here:

http://www.biomedcentral.com/info/publishing_adv.asp
BioMedcentral 